\title{
Modeling tools to estimate the performance of the Thirty Meter Telescope: an integrated approach
}

George Z. Angeli, Jennifer Dunn, Scott C. Roberts, Douglas G. MacMynowski, Anna Segurson, et al.

George Z. Angeli, Jennifer Dunn, Scott C. Roberts, Douglas G. MacMynowski, Anna Segurson, Konstantinos Vogiatzis, Joeleff Fitzsimmons, "Modeling tools to estimate the performance of the Thirty Meter Telescope: an integrated approach," Proc. SPIE 5497, Modeling and Systems Engineering for Astronomy, (16 September 2004); doi: 10.1117/12.552523

SPIE Event: SPIE Astronomical Telescopes + Instrumentation, 2004, Glasgow, United Kingdom 


\title{
Modeling tools to estimate the performance of the Thirty Meter Telescope: an integrated approach
}

\author{
George Z. Angeli ${ }^{1}$, Jennifer Dunn ${ }^{2}$, Scott Roberts ${ }^{2}$, Doug MacMynowski ${ }^{3}$, Anna Segurson ${ }^{1}$ \\ Konstantinos Vogiatzis ${ }^{1}$, Joeleff Fitzsimmons ${ }^{2}$, \\ ${ }^{1}$ New Initiatives Office, AURA Inc. \\ ${ }^{2}$ Herzberg Institute of Astrophysics, National Research Council, Canada \\ ${ }^{3}$ California Institute of Technology
}

\begin{abstract}
A sound system engineering approach and the appropriate tools to support it are essential in achieving the scientific and financial objectives of the Thirty Meter Telescope project. Major elements of the required tool set are those providing estimates for the performance of the telescope. During the last couple of years, the partners in the consortium developed a wide range of modeling and simulation tools with various levels of fidelity and flexibility. There are models available for time domain and frequency domain simulations and analysis, as well as for lower fidelity, parametric investigations of design trade-offs and for high fidelity, integrated modeling of structure, optics and control. Presented are characteristic simulation results using the existing preliminary point designs of the TMT, with emphasis on the telescope performance degradation due to wind buffeting. Under the conditions modeled, the wind induced image jitter and image quality degradation was found comparable to good atmospheric seeing.
\end{abstract}

Keywords: integrated modeling, telescope control, extremely large telescopes

\section{INTRODUCTION}

The Thirty Meter Telescope (TMT) project, pursued by a consortium of California Institute of Technology, University of California, Association of Universities for Research in Astronomy, and Association of Canadian Universities for Research in Astronomy, has the ambitious goal of developing and constructing an extremely large, ground based, segmented mirror telescope. The project raises numerous technical questions that are difficult or impossible to address meaningfully with traditional engineering tools. A few characteristic examples are:

- The impact of wind buffeting on the image quality and pointing stability of the telescope;

- The optimal spatial and temporal bandwidth and stroke for different control systems (primary, secondary, tertiary, and possibly adaptive secondary mirrors);

- The effects of hierarchical and parallel control loop couplings and contentions;

- The impact of chopping on image quality, AO loop stability, and pointing stability;

- The best achievable settling time for slewing and nodding;

- The residual Laser Guide Star jitter due to platform vibrations.

In this early phase of the program, the first question seems to be the most important and urgent, namely (i) if the effects of wind buffeting are threatening the feasibility of the telescope, and (ii) what the difference is in wind sensitivity among the various possible telescope configurations. In order to provide answers to these questions, a dynamic, integrated model of the telescope is required.

To meet the TMT budget without significant compromises on the performance, the project chose to explore technologies that have emerged since the design phase of the former generation of telescopes. One of these emerging technologies is the huge advancement in affordable computing power. The computing power, through the availability of sub-system level modeling tools, for example finite element analysis (FEA), was a major factor 10 to 
15 years ago in the affordability of the 8 meter class telescopes. Today's technology allows us to take the next leap forward, and integrate these tools into an end-to-end integrated model of the telescope. This advanced modeling technique has the potential of reducing the telescope cost in various ways, like:

- Identifying design options with the same performance but reduced sub-system specifications and tolerance requirements;

- Identifying design options with the same performance but reduced sensitivities to environmental effects, resulting relaxed mitigation requirements;

- Eliminating redundant functionalities and controls.

The role of modeling in the design process is twofold: (i) it facilitates the design by defining the mathematical environment in which the design established and tested, and (ii) provides feedback to the design process. A full system model, as shown in Figure 1, can serve the project from the initial system trades and feasibility studies to the final implementation and commissioning of the observatory.

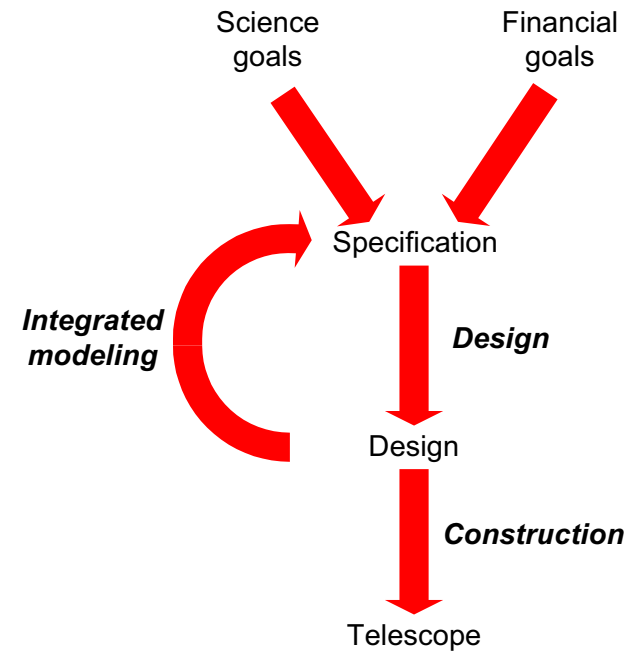

Figure 1 Role of integrated modeling in the system development process
The operation of the TMT cannot be understood; furthermore, its performance cannot be reliably predicted without considering the effects of the various control systems inherent to the success of the telescope. A joint, integrated simulation of structure, optics, and control is necessary because:

- The dynamics of the telescope and control loops are expected to have significant effect on the performance;

- Considerable interactions among the various parallel and hierarchical sub-systems are anticipated.

The integrated modeling tools, developed by the TMT partner institutions, are capable of simulating the dynamic interactions of atmosphere, optical surfaces of the telescope, forces on the structure of the telescope, edge sensors between primary mirror segments, wavefront sensors, and the active control of the secondary mirror and primary mirror segments. They also feature links to adaptive optics simulations.

It is foreseeable that during the development phase of the TMT, and beyond, there will be many versions of the integrated telescope model. These iterations are going to reflect the evolution of the telescope design itself, from various point designs to the final design. They also will reflect the different aspects of a given design to be investigated: tolerance and sensitivity analyses, loop contention analysis, and overall performance estimates. Consequently, it is imperative from both budgetary and scheduling reasons to keep the explicit integrated modeling efforts efficient.

The integrated modeling approach outlined in this paper is combining software packages used by the different engineering groups (e.g., optics, adaptive optics, controls, structures) into a single model. The integration is facilitated by a "glueware" that manages the communications between the sub-system models of the telescope and schedules the execution of these modules.

Fundamental programmatic and technical design decisions will be based on the results of integrated modeling. This fact emphasizes the importance of the verification of the integrated model, which lends credibility to the results. As the project proceeds, experimental prototypes of different sub-systems of the telescope will be available. Since the designs and hence the computer models of these prototypes will be available, these experiments will be the ultimate verification tools for integrated modeling. Until this verification path becomes feasible, the chosen modeling strategy itself provides preliminary, but substantial credibility to the model. The engineering design and modeling 
tools used for designing the telescope sub-systems, and also used in the integrated model, are well-established packages with huge user bases. These software packages were developed by third party software companies, most of them have been on the market for a substantial amount of time. The large user base implies a significant number of cases already verified through actual implementation, but also detailed methodology and a sizeable expert base.

\section{COMPUTATIONAL ENVIRONMENT}

Although the implementation of the integrated modeling tools at the different partner institutions are slightly different, the underlying concepts and basic computational environment are the same: the simulation is usually running in time domain, the core model is coded in MATLAB/Simulink, with links to optical ray trace software (Figure 2).

The modeling environment includes advanced tools, like sophisticated modal reduction of the structural model [1]. There is source code control (CVS) implemented for the developed models and associated software. The simulation tools are capable of running on a cluster of Linux computers (Figure 4), as well as on Windows. The details of the realization are discussed elsewhere [2-5]

It may be necessary to run the integrated simulations many times for high-level trade studies, to adequately explore the parameter space. A parametric, frequency domain version of the modeling tool set can highly improve the speed of calculations. Since it uses simplifying assumptions, the results have lower fidelity. Currently only a parametric wind model is available, but the parameterization of the structural and control model is in progress.

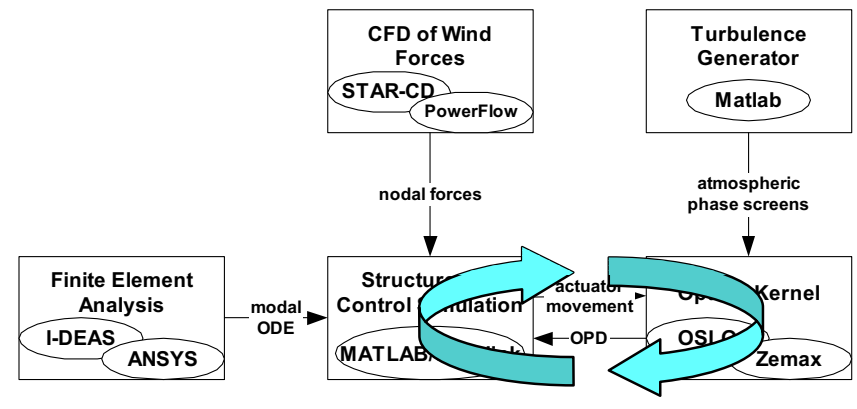

Figure 2 The basic kernel of computations

It is reasonable and feasible to separate the telescope simulation from the process of generating the model. The setup parameters can be changed in a straightforward way; in some realization of the tool set even a GUI is available for configuring the simulation (Figure 3). The Model Generator creates the configuration in an independent process, and saves it into files. When the simulation begins, each block reads its configuration variables and initial values from these files. The Model Generator acts as a translator of external information and processes (Figure 5).

Estimating the performance of the observatory is not complete without approximating the behavior of the science instrument. Although currently the assumption is that the instrument simulation will function as a post-processor to the integrated model (Figure 5), in the future it may become necessary to couple the two simulations due to the potential requirement of optical feedback from the instrument. 


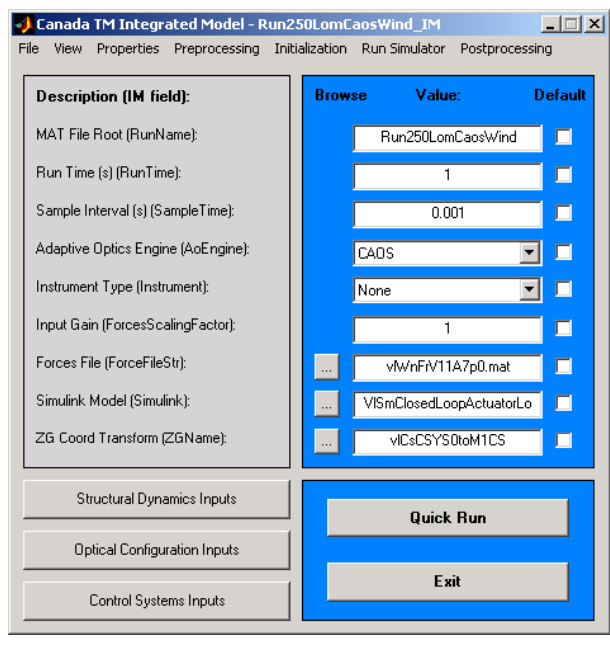

Figure 3 The user interface developed at HIA

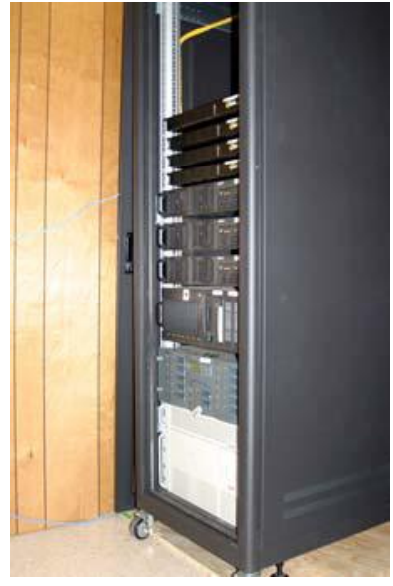

Figure 4 The 16 nodes Beowulf cluster at AURA for integrated modeling and CFD

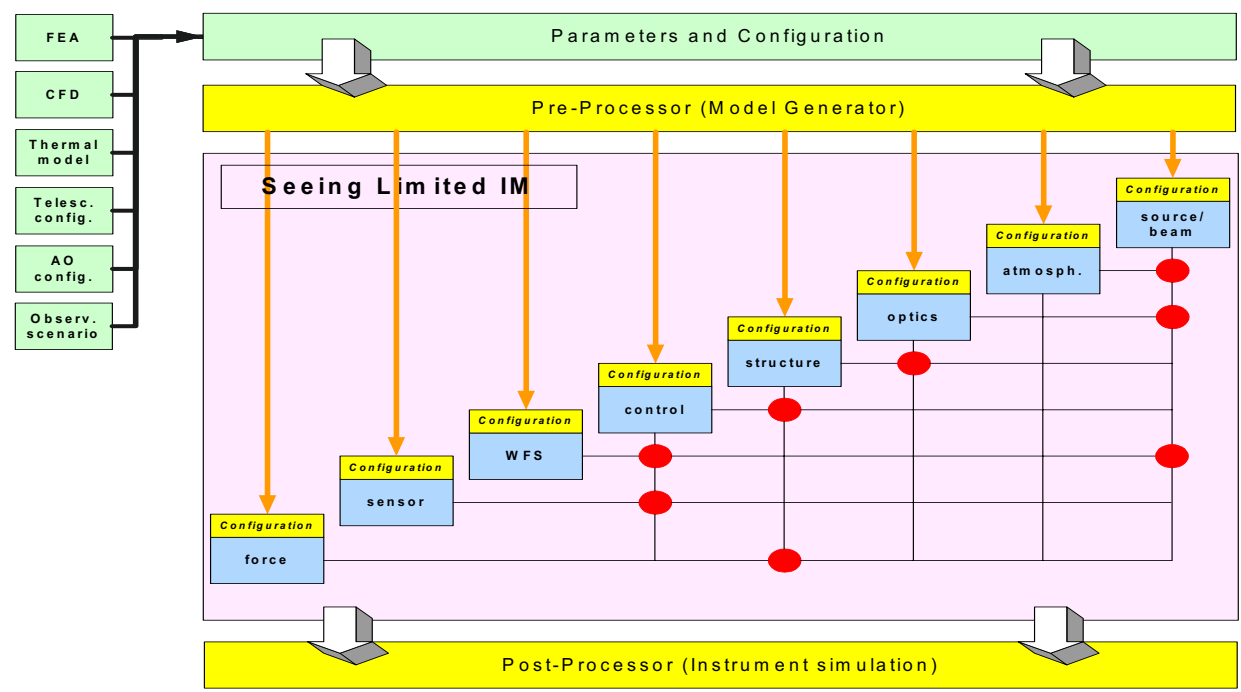

Figure 5 The computational environment of the integrated modeling tools developed by the TMT partners. (Dots represent the internal interfaces connecting the individual simulation modules)

\section{STRUCTURAL MODEL}

The structural model used in our simulations is a linear, time invariant, dynamic system. While the VLOT finite element model is highly detailed, the CELT and GSMT models are rather strawman designs with a few thousand or a few hundred nodes respectively. Consequently, the CELT and GSMT point designs do not contain the segment dynamics. For GSMT simulations, the segment masses are lumped to a single support node for each raft (7 segment block). The individual segments are added in MATLAB as inertialess elements supported by 3 spring actuators with $10 \mathrm{~N} / \mu \mathrm{m}$ stiffness. For the initial feasibility and trade studies evaluating the effect of wind, the lack of segment 
dynamics is not a significant limiting factor, as the resonant frequencies corresponding to segment dynamics would be rather high $(50-70 \mathrm{~Hz})$, relative to the wind spectrum.

The finite element model of the telescope is imported into MATLAB as a set of ordinary differential equation: the uncoupled modal equations of motion of the structure. Currently the tool set is capable of converting ANSYS and IDEAS finite element models.

The architecture of the structural model in Simulink is shown in Figure 6. The physical forces acting on various degrees of freedom of the structure are converted into modal forces, and applied on the state space representation of the structure. During simulations, the core modal differential equations are integrated to obtain the instantaneous modal coefficients. By means of these modal coefficients, the real, physical node displacements are assembled as a linear combination of the mode shapes.

Although the actual simulations are usually carried out in time domain, the model can provide transfer functions between given inputs and outputs to enhance the understanding of telescope behavior. Figure 7 shows not only the structural modes of the telescope, but also their effect on the optical performance. It is visually clear in the figure that the modes involved in exit pupil tip (fringe Zernike coefficient \#1) are mostly different from the ones involved in tilt (fringe Zernike coefficient \#2). It is also obvious that the cross coupling (from Fx to Z2 and Fy to Z1) is negligible, compared to the direct effects. As the structural damping is limited (assumed $2 \%$ in the current model), the structural modes remain quite pronounced even with high control bandwidth.

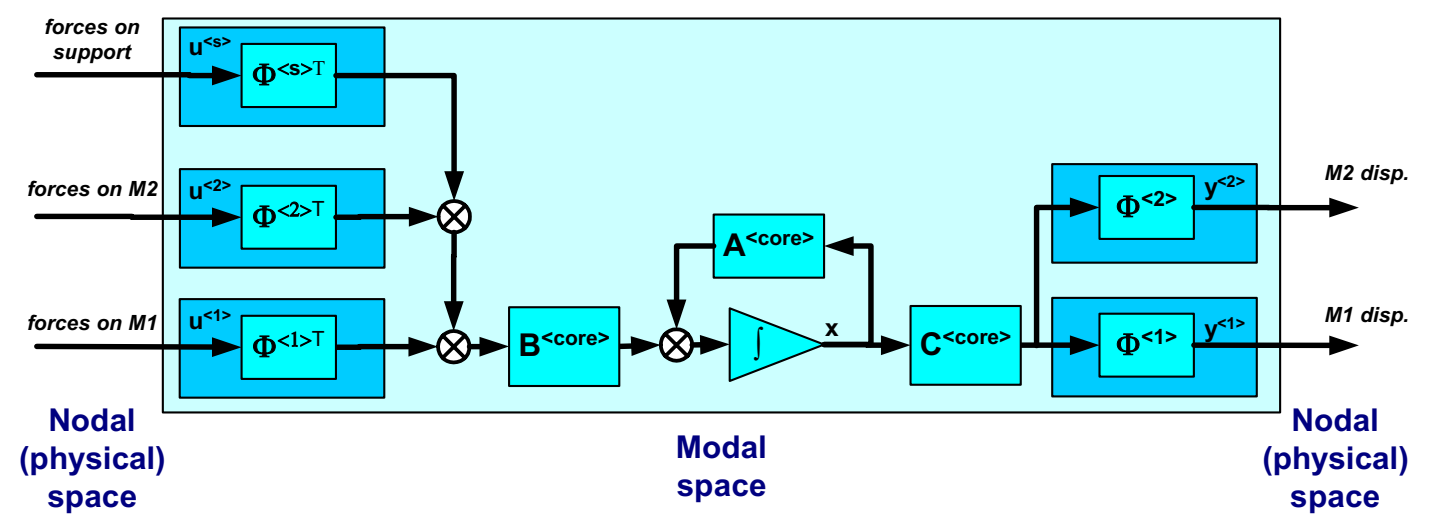

Figure 6 The outline of the model for telescope structure, as it calculated in MATLAB/Simulink

To achieve the required performance, the deformation of the telescope should be controlled. In the simulations reported, two distinct control systems are applied. The mechanical feedback loop features edge sensors detecting the steps between adjacent segments. Some of the sensors considered can also measure the dihedral angle. Detecting the dihedral angle reduces the unobservable primary mirror modes from four to three: the $\mathrm{x}$ and $\mathrm{y}$ tilts and piston. However, the current model is conservative in the sense that it can observe only steps perpendicular to the optical surface. A control bandwidth of $2 \mathrm{~Hz}$ was chosen for the edge sensor loop.

The other control implemented is a basic optical feedback loop detecting the exit pupil tip/tilt, and correcting it by tilting the secondary mirror. As the current objective of the simulations is not control system design or verification, but the evaluation of wind effects, the bandwidth of this loops is set to rather high, $100 \mathrm{~Hz}$. The result is an almost complete elimination of image jitter, which allows the examination of higher order aberrations. If, in reality, such a high control bandwidth would become necessary, the tip/tilt correction will probably be implemented either by a fast steering mirror or a deformable secondary mirror face-sheet.

In the current GSMT simulations, both control loops are decoupled from the structural dynamics, i.e. the actuators modeled are position actuators instead of force actuators. It is rather a choice than a limitation of the tool set, as force actuators were successfully implemented in earlier versions of the integrated model $[5,6]$. 

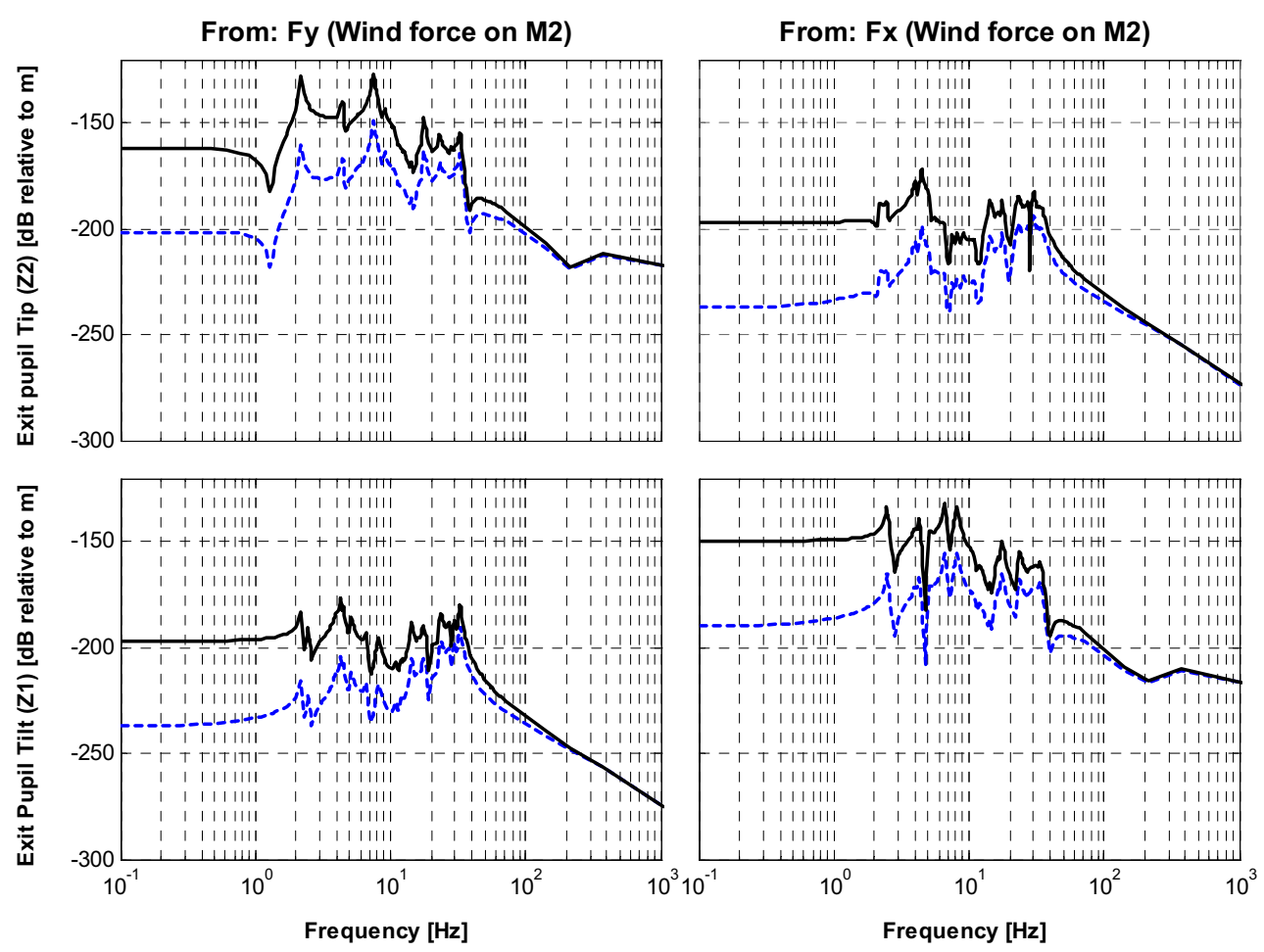

Figure 7 System transfer functions from the wind forces applied on the secondary mirror to image jitter (exit pupil OPD tip/tilt expressed as fringe Zernike coefficients). The dotted lines show the effect of an optical feedback controlling secondary mirror tip/tilt. The exit pupil OPD was estimated by a Zernike based linear optical model (see Section 5).

Unless otherwise noted, the results presented in this paper were derived by using the GSMT structural and optical models, in order to avoid potential confusion due to the slightly different behavior of the point designs (VLOT, CELT, and GSMT).

\section{WIND MODEL}

It is generally expected that the major disturbance to an extremely large telescope is the wind shaking and buffeting. In order to gain a better understanding of the wind effects in and around the telescope dome, the TMT partner institutions carried out extensive wind tunnel measurements and computational fluid dynamics (CFD) analyses [79]. Figure 8 shows the wind tunnel for the Canadian VLOT telescope in its Calotte type dome. A similar setup with generic ELT dome and the GSMT telescope was measured in the Caltech wind tunnel. The wind tunnel measurements verified the CFD techniques used at AURA and IAR in Canada [8,9].

The telescope surface pressure data provided by CFD were converted to forces acting on the telescope structure and primary mirror [3]. The pressure given on the CFD grid was re-sampled on the structural node grid, forces were calculated by assigning appropriate surfaces to the nodes, and the forces then applied to the corresponding nodes.

In the configuration studied in this paper the telescope is facing the wind and pointed to 30 zenith angle, as it is shown in Figure 9, There are high pressure gradients around the secondary mirror, both in the wind direction (y in telescope coordinate system), and in the optical axis of the telescope (z). 


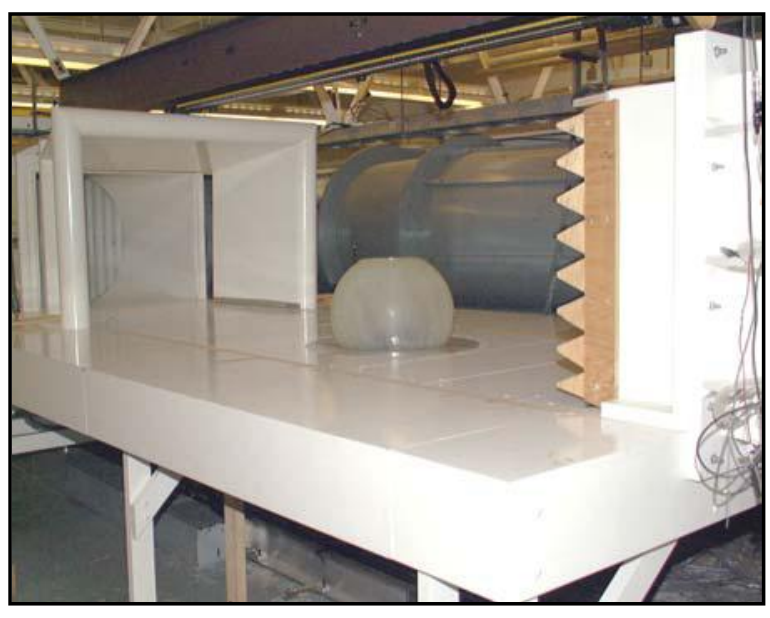

Figure 8 The model of VLOT (HIA) and its dome in the wind tunnel at IAR (Canada)
This high pressure gradients result in significant forces acting on the secondary mirror both in $y$ and $\mathrm{z}$ direction. The corresponding $\mathrm{y}$ and $\mathrm{z}$ displacements of the secondary mirror dominate the rigid body motion of the mirror (see Figure 10). The pressure snapshot in Figure 9 also clearly shows the vortex shedding at the observing slit.

The PSD curves in Figure 10 illustrate the structural modes excited by wind forces. For y de-center, it is the lowest rocking mode of the elevation structure $(2.17 \mathrm{~Hz})$, while for $\mathrm{z}$ piston, it is a torsion-piston mode of the secondary mirror support structure (5.4 $\mathrm{Hz}$ ).

The wind pressure inside the dome is dominated by the very low frequency acoustic waves generated by the shear layer modes in the observing slit. As they have long wavelength, the corresponding correlation length is also significantly larger than the enclosure size, which explains the low pressure gradient on the primary mirror. However, the Gemini South wind measurements do not indicate the presence of significant tonal pressure components in the dome $[10,11]$. The only sign of these shear layer modes is a barely visible "hump" in some of the pressure data recorded in a closed dome.

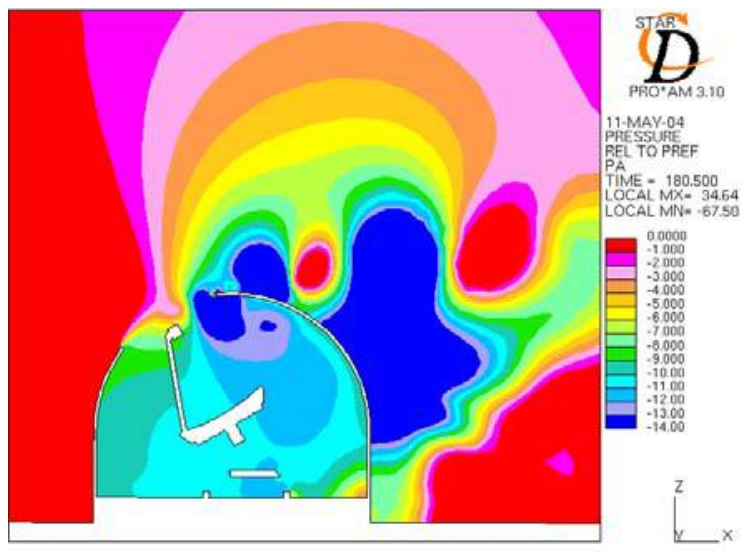

Figure 9 A snapshot of the pressure field in and around the generic ELT dome with a GSMT like telescope inside, as it was provided by full scale CFD at NIO
Our assumption was that small spatial frequency structural features always present on a real telescope dome, together with the particularly large, open wind vents on the Gemini dome prevent wind energy accumulation in the shear layer modes. To test this hypothesis, we carried out both wind tunnel tests and CFD analyses with a vented dome (Figure 11). Indeed, as Figure 12 demonstrates, opening vents on the side of the dome reduces the effect of the shear layer modes.

The deformation of the primary mirror can be expressed in different orthogonal basis sets. One of these are defined by the singular value decomposition of the edge sensor influence function (see Figure 13) $[6,12]$

Another usual way of characterizing optical surfaces is the Zernike decomposition of their shape (Figure 14). (In this paper, we use fringe (University of Arizona) Zernike terms normalized to the largest value of the given term.) Both decomposition show the same characteristic, namely most of the wind energy is concentrated in the lowest order, smoothest modes. This result is consistent with earlier simulation results obtained with different wind models $[6,13]$. 


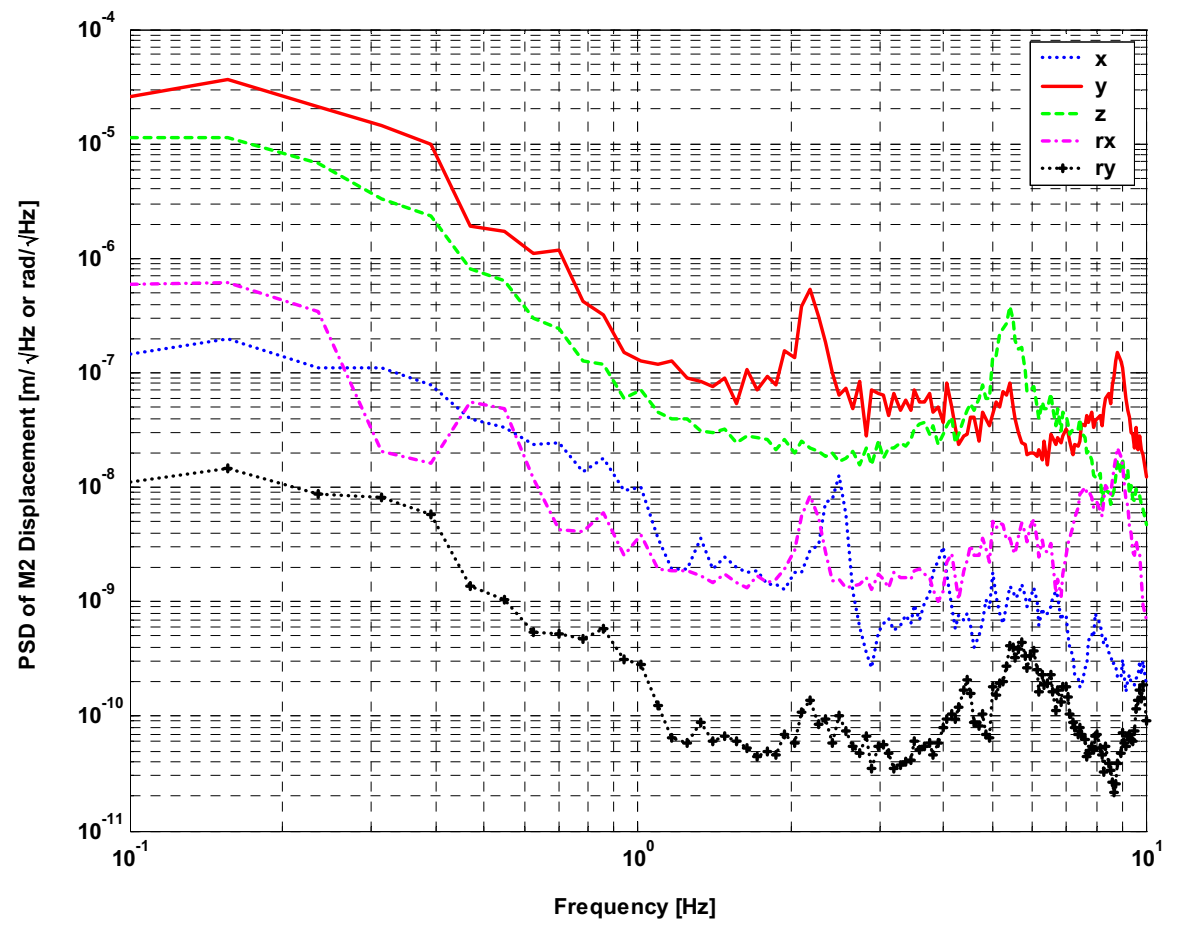

Figure 10 The PSD of secondary mirror displacements due to wind load in non-vented dome. As the radius of the secondary mirror is 1 meter, the tilt in radian corresponds to fringe Zernike tilt coefficient in meter (not considering the sign and ordering).

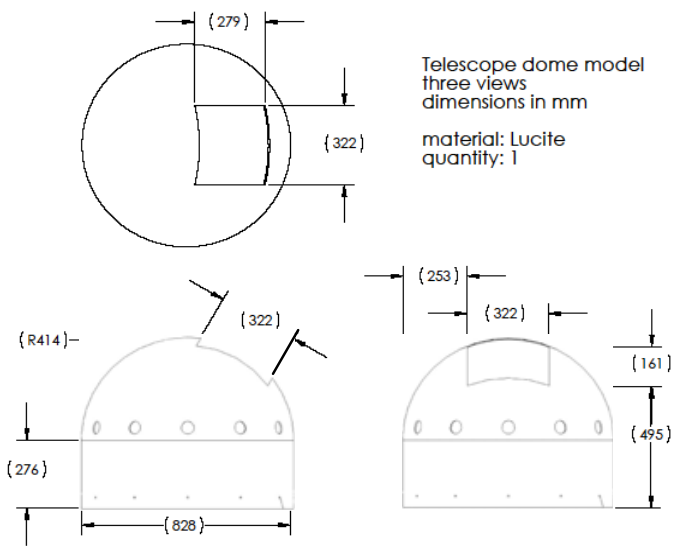

Figure 11 The model of the generic ELT dome used in the Caltech wind tunnel. It clearly shows the circular vents on the side of the dome

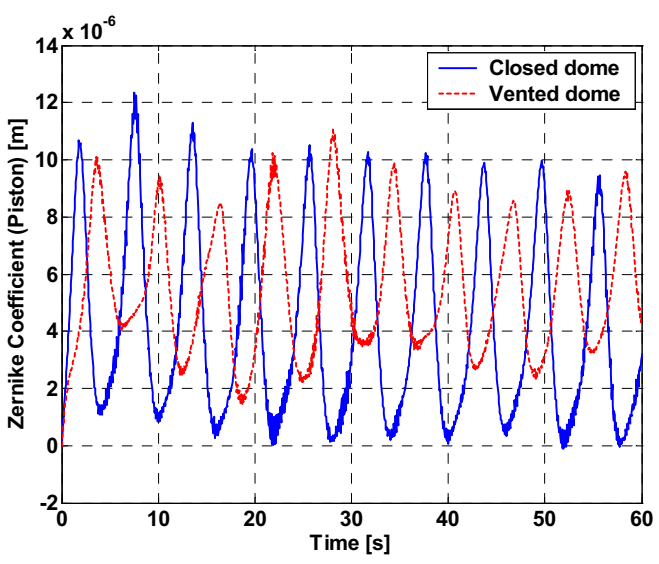

Figure 12 Time history of the primary mirror piston motion due to the wind load in vented and non-vented dome

For many applications, the continuity of the primary mirror is even more important than its shape. A good overall measure of this continuity is the ensemble average of the temporal RMS of the individual edge sensor readings. Furthermore, the ensemble average PSD of these readings even show the frequency distribution of the RMS values. Figure 15 reveals that in the case modeled the dominant source of mirror discontinuity is the shear layer tone. The dashed line shows the effectiveness of a simple edge sensor loop. 
These effects are even more appreciable in the cumulative RMS plots of Figure 16. While the open loop discontinuity reaches $100 \mathrm{~nm}$, the edge control loop reduces it to the more acceptable level of $20 \mathrm{~nm}$

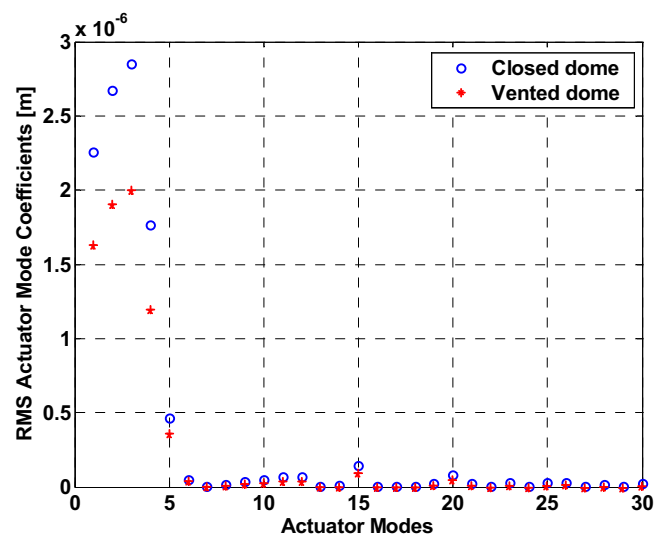

Figure 13 Actuator mode decomposition of the primary mirror deformation due to wind load in vented and non-vented domes (temporal RMS of the actuator mode coefficients). The actuator modes are normalized to 1 meter

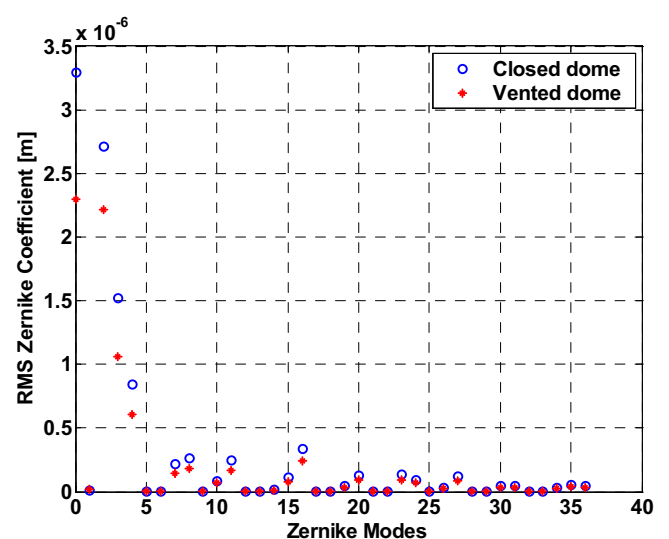

Figure 14 Fringe Zernike decomposition of the primary mirror deformation due to wind load in vented and non-vented domes (temporal RMS of the Zernike coefficients)

The simulations described above require individual CFD runs for each configuration modeled. This procedure is highly computationally intensive and time consuming, if the objective is the exploration of a large parameter field to support high-level system trades. However, simplifying assumptions can lead to a parametric wind model representing our current understanding of the wind inside and around the telescope dome [14]. The major assumption behind of such a model is the proposition of linearity, namely the flow field is the linear combination of two, in this approximation independent, flow fields:

- The acoustic fields generated by the shear layer modes at the observing slit, defined by the dimensions of the slit, its position relative to wind direction, and the external wind speed;

- The broadband well developed von Karman turbulence fields of the air flowing through the openings of the dome, with an outer scale defined by the characteristic dimension of the opening.

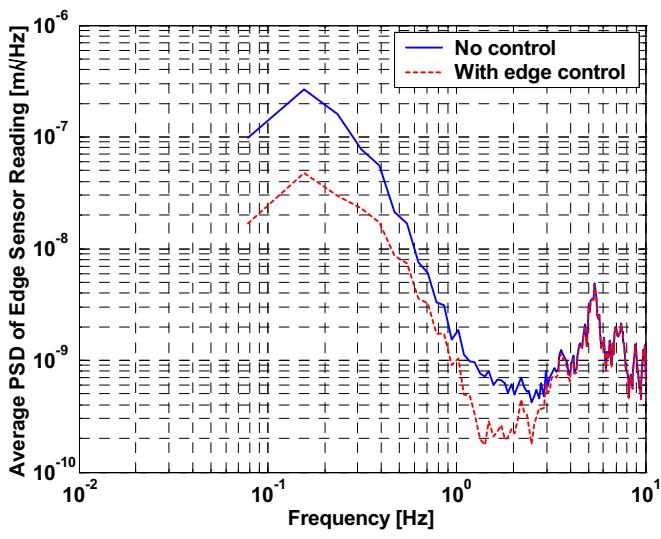

Figure 15 The ensemble average of the PSD calculated for each individual edge sensor reading. The dashed line shows the effect of edge control loop. The PSD was calculated after the mean value was removed.(unvented dome)

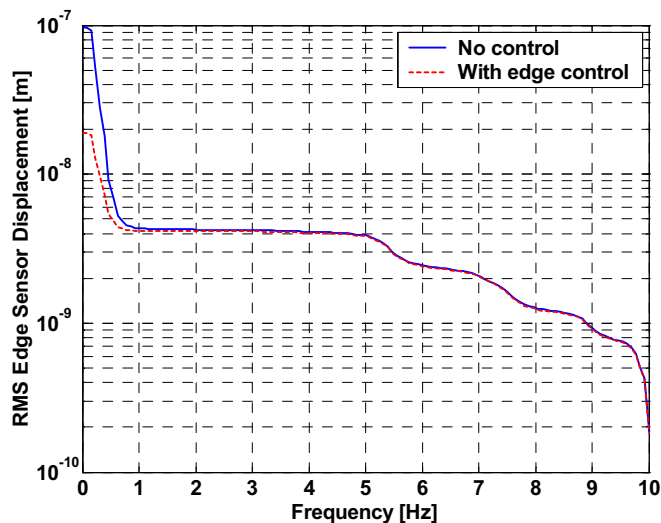

Figure 16 The integral of the PSD in Figure 15 at/above a given frequency, providing the ensemble average RMS edge sensor reading above that frequency.(unvented dome) 


\section{OPTICAL MODEL}

The segments of the primary mirror are nominally hexagons. However, as a paraboloid surface cannot be covered with true hexagons, the mirror segments are hexagonal in projection only. The VLOT design projects the segments on a spherical surface, while the GSMT point design uses a plane perpendicular to the optical axis as reference. Both designs allow segment tip, tilt, and piston displacements, but not de-centers and clocking. Although this limitation is certainly too restrictive for thermal or gravitational analyses, it is assumed that the dynamic wind forces do not introduce measurable de-centers and clocking. The current models also neglect the higher order surface errors on the segments, like support print throughs.

Five degrees of freedom of the secondary mirror rigid body motion are considered in the optical module of the integrated models reported here, with the rotation about the optical axis neglected.

The communication between the optical and structural modules is based on the virtual telescope concept. Independent telescope models are built in the structural and optical environments of the simulation. The models are generated by the same algorithm, in the same global coordinate system. In each time step, the models are synchronized by communicating the low order Zernike coefficients, or rigid body motion components of each optical surface (Figure 17).

The optical module can feature four different optical kernels:

- $\quad$ Ray tracing in OSLO, with up to 1000 by 1000 rays resolution, which translates to 3 cm resolution [3,4];

- $\quad$ Ray tracing in Zemax, with up to 256 by 256 rays resolution [15];

- Full linear optical model linking the exit pupil OPD grid to the displacements of telescope nodes [15];

- Zernike based linear optical model linking the Zernike coefficients of the exit pupil OPD to the Zernike coefficients of the mirror deformations [16].

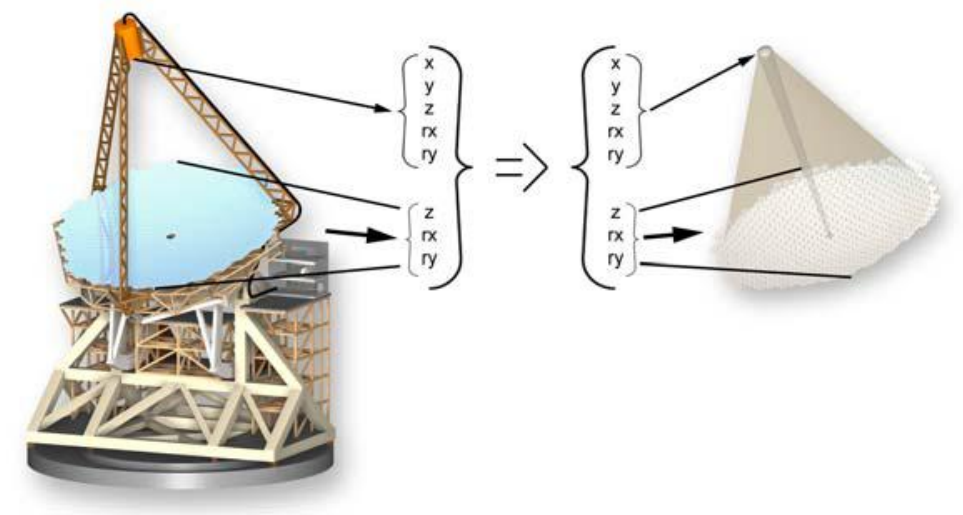

Figure 17 The virtual telescope concept, calling for independent, but identical telescope models built in MATLAB and OSLO. The rigid body displacements, or, alternatively, the Zernike coefficients (extended with the de-centers) of the optical surfaces are communicated between the two virtual telescopes.

The linear optical models are pre-calculated and used in lower fidelity simulation, in order to improve the speed of the calculations. The Zernike based model can also help in quick sanity checks and mental visualization of the telescope performance.

The OSLO ray trace kernel is set up on Linux. The communication with MATLAB, also running on Linux, is facilitated by sockets. The socket communication enables fast transfer of large amounts of data, like complete OPD 
maps, from OSLO to MATLAB [3]. OSLO also supports the linking of $\mathrm{C}$ language routines containing the user defined surfaces and custom ray traces.

The optical module uses two realizations of Brent Ellerbroek's Cerro Pachon atmospheric profile to simulate seeing effects [17]. One realization calculates the time series of atmospheric phase screens at the entrance pupil of the telescope in MATLAB using a sub-section of TAOS, and introduces it to the ray trace engine as a diffractive surface. The other realization uses the Arroyo AO code written in $\mathrm{C}$ to calculate the atmospheric phase screen at the entrance pupil.

The telescope integrated model also provides links to CAOS AO simulation tool. The details of this link are described elsewhere [18]. Once the optical module is integrated into the model, it becomes capable of predicting the performance of the telescope. The following results use a 1-micron wavelength. A major performance metric is the diameter that encircles $80 \%$ of the energy at the focal plane (Figure 18). In our calculations, the center of the circle was always repositioned to the instantaneous centroid of the image, and the light energy was integrated for a full minute

The curves in Figure 18 show that the wind induced image degradation is below the atmospheric seeing. It is clear that the edge sensor control loop, besides keeping the primary mirror continuous, also partially restores its shape, thus reducing the encircled energy radius by a factor of two.

As the encircled energy does not contain the image jitter, it is investigated separately. Figure 19 and Figure 20 show the wind induced image jitter

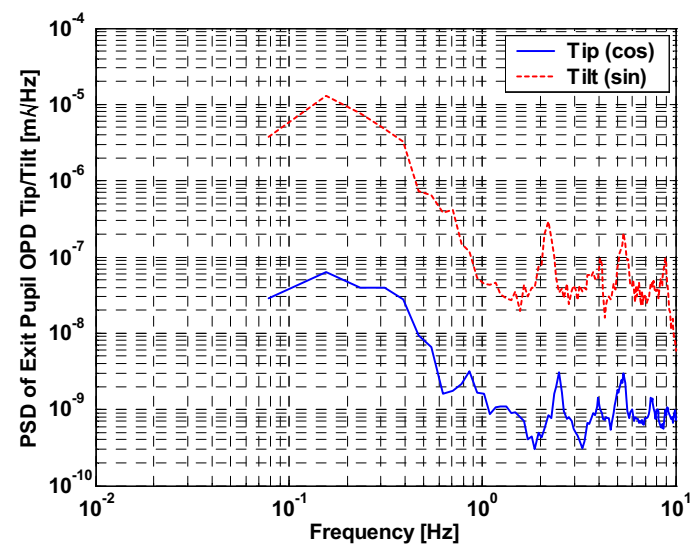

Figure 19 PSD of exit pupil OPD tip/tilt, expressed as fringe Zernike coefficients Z1 (cos) and Z2 (sin).

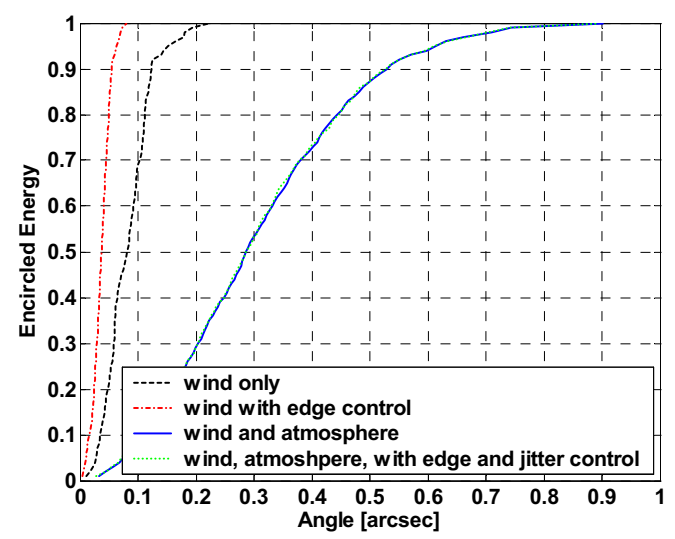

Figure 18 The encircled energy averaged over a minute. As it is referenced to the centroid of the image, it does not contain image jitter (unvented dome)

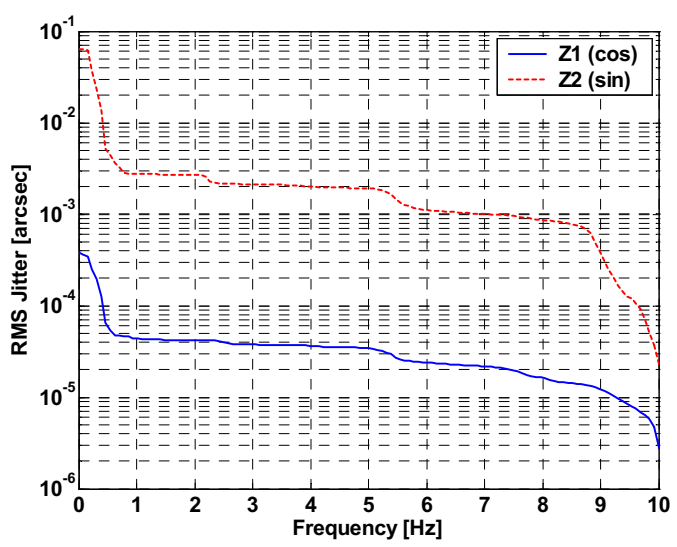

Figure 20 The integral of the PSD in Figure 19 at/above a given frequency, providing the RMS image jitter above that frequency 


\section{CONCLUSIONS}

The simulations show that under the conditions modeled, wind induced image degradation of a 30 meter class telescope is not negligible. However, data also suggest that proper dome design and appropriate control loops can mitigate the effect of wind.

For the wind load applied in the current simulations, the edge sensor based active optics for the primary mirror can reduce the edge displacements between segments below specification levels $(25 \mathrm{~nm})$. It is worth noting, though, that the applied wind is not necessarily worst case, as it is dominated by the acoustic waves of the shear layer modes with correlation lengths much larger than the segment size. Gemini South wind measurements indicate a different pattern, dominated by broadband von Karman turbulence with much shorter correlation length. However, earlier simulations of the same structure using a wind model based on the Gemini measurements also concluded that a proper edge control system could mitigate the wind induced primary mirror discontinuities.

Both dynamic image degradation and image jitter due to wind buffeting are found to be below the natural seeing. However, for open loop these errors still constitute a significant, and probably intolerably high, portion of the telescope error budget. Although the edge sensors cannot detect the first four primary mirror modes, the effect of the edge control loop is significant in image correction, as the models predict it is capable of reducing wind seeing by a factor of two.

As we saw earlier, dome vents can significantly reduce the strength of the shear layer modes. Unfortunately, the venting holes increase the broadband component of wind. By developing a parametric wind model, the different components of wind become individually tunable, which in turn may lead to a more realistic wind load estimate for the telescope.

For the immediate future, the most important application of the developed integrated modeling tool set is supporting the high level system trades of the Thirty Meter Telescope project: the choice of elevation axis position, secondary mirror type and size. By simulating the different structural and optical point designs, the tool set will provide valuable performance and sensitivity estimates for the selection process.

\section{ACKNOWLEDGEMENTS}

The authors would like to thank Robert Upton of AURA New Initiatives Office for his valuable suggestions and comments. We are also grateful to Brent Ellerbroek and Myung Cho of AURA New Initiatives Office for their reviews and comments.

The authors present this paper as part of the work of the Thirty Meter Telescope (TMT) Project. TMT is a partnership of the Association of Universities for Research in Astronomy (AURA), the Association of Canadian Universities for Research in Astronomy (ACURA), the California Institute Of Technology and the University of California. The partners gratefully acknowledge the support of the Gordon and Betty Moore Foundation, the US National Science Foundation, the National Research Council of Canada, the Natural Sciences and Engineering Research Council of Canada, and the Gemini Partnership.

The New Initiatives Office is a partnership between two divisions of the Association of Universities for Research in Astronomy (AURA), Inc.: the National Optical Astronomy Observatory (NOAO) and the Gemini Observatory.

NOAO is operated by AURA under cooperative agreement with the National Science Foundation (NSF). 
The Gemini Observatory is operated by AURA under a cooperative agreement with the NSF on behalf of the Gemini partnership: the National Science Foundation (United States), the Particle Physics and Astronomy Research Council (United Kingdom), the National Research Council (Canada), CONICYT (Chile), the Australian Research Council (Australia), CNPq (Brazil) and CONICET (Argentina)

\section{REFERENCES}

1. R. Yu, S. Roberts, and I. Sharf, "Model order reduction of structural dynamics of a Very Large Optical Telescope," Proceedings of SPIE 5497, 72 (2004).

2. J. Dunn, S. Roberts, J. Fitzsimmons, G. Herriot, J. Pazder, M. Smith, and J.-P. Verna, "VLOT/TMT integrated model enhancements," Proceedings of SPIE 5497, 58 (2004).

3. A. Segurson and G. Z. Angeli, "Computationally efficient performance simulations for a thirty mater telescope (TMT) point design," Proceedings of SPIE 5497, 33 (2004).

4. G. Z. Angeli, A. Segurson, R. Upton, B. Gregory, and M. Cho, "Integrated modeling tools for large ground based optical telescopes," Proceedings of SPIE 5178, 49-63 (2003).

5. S. Roberts, J. Pazder, J. Fitzsimmons, G. Herriot, N. Loewen, M. Smith, J. Dunn, and L. Saddlemyer, "Integrated modeling of the Canadian Very Large Optical Telescope," Proceedings of SPIE (2003).

6. G. Z. Angeli, R. Upton, A. Segurson, and B. L. Ellerbroek, "Active optics challenges of a thirty meter segmented mirror telescope," Proceedings of SPIE (2003).

7. T. S. Pottebaum and D. G. MacMynowski, "Wind tunnel testing of a generic telescope enclosure," Proceedings of SPIE 5495, 30 (2004).

8. J. Fitzsimmons, J. Dunn, G. Herriot, L. Jolissaint, S. Roberts, M. Mamou, and K. Cooper, "Predicting the aerodynamic performance of the Canadian Very Large Optical Telescope," Proceedings of SPIE 5497, 31 (2004).

9. K. Vogiatzis, A. Segurson, and G. Z. Angeli, "Estimating the effect of wind loading on extremely large telescope performance using computational fluid dynamics," Proceedings of SPIE 5497, 30 (2004).

10. G. Z. Angeli, M. Cho, M. Sheehan, and L. Stepp, "Characterization of wind loading of telescopes," Proceedings of SPIE 4757, 72-83 (2002).

11. M. Cho, L. Stepp, G. Z. Angeli, and D. Smith, "Wind loading of large telescopes," Proceedings of SPIE 4837, 352-367 (2003).

12. D. G. MacMartin and G. Chanan, "Measurement accuracy in control of segmented-mirror telescopes," Applied Optics 43, 608-615 (2004).

13. G. Z. Angeli, M. Cho, and M. S. Whorton, "Active optics and control architecture for a Giant Segmented Mirror Telescope," Proceedings of SPIE 4840, 129-139 (2003). 
14. D. G. MacMynowski, G. Z. Angeli, K. Vogiatzis, and J. Fitzsimmons, "Parametric modeling and control of telescope wind induced vibration," Proceedings of SPIE 5497, 26 (2004).

15. J. Pazder, S. Roberts, M. Smith, J.-F. Lavigne, and H. McKenzie, "The optical modeling tools for the Canadian Very Large Optical Telescope integrated model," Proceedings of SPIE (2003).

16. G. Z. Angeli and B. Gregory, "Linear optical model for a large ground based telescope," Proceedings of SPIE 5178, 64-73 (2003).

17. B. L. Ellerbroek, Journal of the Optical Society of America 19, 1803 (2002).

18. M. Smith, E. Steinbring, J.-P. Veran, G. Herriot, J. Dunn, and S. Roberts, "Integrating MATLAB and IDL: adding adaptive optics to the TMT/VLOT integrated model," Proceedings of SPIE 5497, (2004). 